\title{
Secondary causes of nonalcoholic fatty liver disease
}

\author{
Jacob M. Kneeman, Joseph Misdraji and Kathleen E. Corey
}

\begin{abstract}
Nonalcoholic fatty liver disease (NAFLD) is becoming the most common cause of chronic liver disease in the developing world, found in $17-30 \%$ of the population in Western countries and $2-4 \%$ worldwide. Defined as the accumulation of fatty acid content greater than $5 \%$ of liver weight, NAFLD is a spectrum of disease ranging from simple steatosis to nonalcoholic steatohepatitis. The pathophysiology of NAFLD involves increased de novo synthesis of fatty acids in hepatocytes, the retention of lipids due to impaired hepatocyte apolipoprotein secretion or beta-oxidation. The well-known primary causes of NAFLD are obesity, type II diabetes, dyslipidemia, and insulin resistance. However, other less common conditions can cause a similar clinical and histologic picture, and should be considered in patients who present with NAFLD but do not have traditional risk factors. In this review, we discuss uncommon but important causes of NAFLD, including inborn errors of metabolism, iatrogenic causes, viral hepatitis, and nutritional disorders to provide practicing clinicians with an understanding of the less well recognized causes of NAFLD.
\end{abstract}

Keywords: fatty liver, nonalcoholic fatty liver disease, nonalcoholic steatohepatitis, secondary causes

\section{Introduction}

Nonalcoholic fatty liver disease (NAFLD) is most commonly the result of the impact of the metabolic syndrome on hepatic metabolism. However, in an important minority of cases NAFLD results from specific secondary causes. The goal of this review is to highlight these rare etiologies of NAFLD and provide the clinician with a framework for evaluating these disease etiologies.

\section{Definition}

NAFLD is the accumulation of triglycerides within hepatocytes that exceeds $5 \%$ of liver weight. NAFLD includes a spectrum of disease from simple steatosis to nonalcoholic steatohepatitis (NASH), which can progress to cirrhosis and hepatocellular carcinoma. Histologically, NASH is defined by the presence of macrovesicular steatosis, lobular inflammation, and hepatocellular ballooning. The pathology is often indistinguishable from alcoholic fatty liver disease; therefore, the diagnosis can only be made in the absence of significant alcohol use, defined as less than $30 \mathrm{~g} /$ day for men and $20 \mathrm{~g} /$ day for women [Sanyal et al. 2011].

\section{Incidence}

NAFLD is gradually becoming one of the most prevalent liver diseases today, identified on imaging in $20-33 \%$ of adults. NASH is diagnosed in $3-16 \%$ of potential liver donors in Europe and the United States. It is also a frequent cause of cirrhosis and is projected to be the leading indication for liver transplantation in the US by 2020 [Ratziu et al. 2010; Sanyal et al. 2011].

\section{Demographics}

NAFLD is diagnosed predominantly in the fourth through sixth decades of life, although the childhood obesity epidemic has caused an increase in the rate of pediatric NAFLD. The prevalence of NAFLD varies by ethnicity, affecting $\sim 45 \%$ of Hispanics, $33 \%$ of Whites, and $24 \%$ of Blacks. Among Whites, NAFLD is more common in men than in women, but is equally distributed between
Ther Adv Gastroenterol

(2012) 5(3) 199-207

DOI: 10.1177/

$1756283 \times 11430859$

(c) The Author(s), 2011. Reprints and permissions: http://www.sagepub.co.uk/ journalsPermissions.nav
Correspondence to: Kathleen E. Corey, MD, MPH

Massachusetts General Hospital, 55 Fruit Street, BLK 4, Boston, MA 02114-2696, USA kcoreyapartners.org Jacob M. Kneeman, BA Gastrointestinal Unit, Massachusetts Genera Hospital, Boston, MA, USA Joseph Misdraji, MD Department of Pathology, Massachusetts General Hospital and Harvard Medical School, Boston, MA, USA 
Table 1. Uncommon causes of NAFLD.

\begin{tabular}{ll}
\hline Disorders of lipid metabolism & Medications \\
Abetalipoproteinemia & amiodarone \\
Hypobetalipoproteinemia & tamoxifen \\
Familial combined hyperlipidemia & methotrexate \\
Glycogen storage disease & corticosteroids \\
Weber-Christian syndrome & HAART \\
Lipodystrophy & Starvation \\
Total parenteral nutrition & Wilsons disease \\
Hepatitis C infection & Environmental toxicity \\
Severe surgical weight loss & Celiac disease \\
\hline HAART, highly active antiretroviral therapy; NAFLD, nonalcoholic fatty liver disease.
\end{tabular}

men and women among African Americans and Hispanic adults [Angulo, 2007].

\section{Clinical features}

NAFLD is largely asymptomatic although fatigue and right upper quadrant abdominal pain are reported by a proportion of patients [Day, 2011]. NAFLD is suspected by identifying commonly associated risk factors, finding steatosis on imaging, and excluding other causes of liver disease. Currently, definitive diagnosis can only be made with liver biopsy. Patients can present with either hepatocellular orcholestatic injury. An increasing number of studies have found NAFLD in patients with normal LFTs [Angulo, 2007]. Other laboratory findings may include low titer concentrations of anti-nuclear antibodies, anti-smooth muscle antibodies, and increased ferritin [NeuschwanderTetri, 2005].

\section{Causes of NAFLD}

The primary risk factors of NAFLD are obesity, type II diabetes, and the metabolic syndrome including dyslipidemia and hypertension [Angulo, 2007]. However, diseases other than the metabolic syndrome can be associated with hepatic fat, and these might enter into the differential diagnosis of fatty liver disease of usual type. Some of these have specific clinical and pathologic features that make their distinction from NAFLD straightforward, but others are infrequent and easily overlooked. The emphasis of this review will be on these less common causes of hepatic steatosis and steatohepatitis in order to provide practicing clinicians with a resource for the full evaluation of patients with presumed NAFLD without the common risk factors (Table 1).

\section{Disorders of lipid metabolism}

A number of rare disorders of lipid metabolism have been associated with the development of NAFLD (Table 2).

\section{Abetalipoproteinemia (Bassen-Kornzweig syndromel}

Abetalipoproteinemia (ABL) is an autosomal recessive disorder caused by a mutation in the gene encoding the microsomal triglyceride transfer (MTTP) protein. MTTP is an endoplasmic reticulum protein that transfers triglycerides to apolipoprotein B-100 (apoB100) in the hepatocyte and apolipoprotein B-48 (apoB48) in the enterocyte. The mutation in MTTP prevents apoB100 and apoB48 from combining with triglycerides to form very-low-density lipoproteins (VLDL) and chylomicrons, respectively [Zeissig et al. 2010]. The absence of functioning chylomicrons inhibits effective lipid absorption in the intestines leading to hypolipidemia, fat malabsorption, and neurologic disorders. The absence of VLDL results in the accumulation of triglycerides within hepatocytes and results in significant steatosis. Symptoms are improved with low-fat diet, fat-soluble vitamin supplementation, and, if needed, total parenteral nutrition (TPN).

\section{Hypobetalipoproteinemia}

Familial hypobetalipoproteinemia (FHBL) is an autosomal recessive disorder of lipid metabolism that results in lowered levels of low-density lipoprotein (LDL) and apolipoprotein B. A missense mutation in the apoB100 gene results in an inability of the liver to synthesize VLDL [Harada et al. 2009]. The impaired synthesis and exportation of VLDL causes triglycerides to accumulate in the 
liver, resulting in macrovesicular steatosis. The clinical features of FHBL are similar to those of $\mathrm{ABL}$, with patients presenting with failure to thrive, steatorrhea, and neurologic impairments such as spinocerebellar degenerative ataxia. The standard treatment involves fat-restricted diets and fat-soluble vitamin supplementation [Linton et al. 1993].

\section{Familial combined hyperlipidemia}

Familial combined hyperlipidemia (FCHL) is an autosomal dominant lipid disorder caused by the overproduction of apoB-100. This overproduction leads to increased VLDL production in the liver and increased hepatic and peripheral lipid levels. NAFLD is seen in approximately $75 \%$ of FCHL patients [de Bruin et al. 2004]. Lifestyle changes including low-fat diets, increased exercise, weight loss, and smoking cessation in conjunction with HMG CoA reductase inhibitors reduce the characteristic hyperlipidemia [Rubenfire et al. 2010].

\section{Glycogen storage disease}

Glycogen storage diseases (GSDs) are disorders of glycogen metabolism that result in abnormal storage of glycogen. While 12 forms of GSD have been described, types 0, I, III IV, VI and phosphorylase $\mathrm{b}$ kinase deficiency are most commonly associated with liver disease [Burwinkel et al. 2003; Kishnani et al. 2010]. Glycogen is stored in the liver and released during fasting to tissues unable to synthesize glucose. Both hepatomegaly and hypoglycemia result from altered liver glycogen metabolism. Patients frequently present early in life with growth retardation, lactic acidosis, and development delay. Adults with GSD are at risk of progression of their liver disease to cirrhosis and of the development of hepatic adenoma [Reddy et al. 2007]. Treatment involves avoidance of fasting, ingestion of corn starch (a slowly absorbed form of glucose) and in some cases, liver transplantation [Muraca et al. 2002].

\section{Weber-Christian disease}

Weber-Christian disease is a nodular, nonsuppurative form of panniculitis associated with abnormal metabolism of fat [Allard, 2002]. Patients typically present with fever, arthralgias, myalgias, skin lesions, and painful subcutaneous nodules on the extremities. Fatty infiltration of the liver is frequently identified with macrovesicular steatosis and Mallory-Denk bodies [Kennedy and Murphy, 1949; Wasserman et al. 2001]. The etiology of the disease remains unknown and immunosuppressive regimens have achieved limited success.

\section{Lipodystrophy}

Lipodystrophy is characterized by the abnormal redistribution of adipose tissue in the body, or even its complete loss (i.e. lipoatrophy). Congenital generalized lipodystrophy is an autosomal recessive disorder with a frequency of 1 in 10 million. Severe fat loss, voracious appetite, accelerated linear growth, and advanced bone age are clinical features of infants with this syndrome. Acquired lipodystrophy starts early in childhood or later in adolescence in previously healthy individuals. Another cause of acquired lipodystrophy is the chronic use of nucleoside analogs or highly active antiretroviral therapy (HAART) to treat patients with HIV. Complications of acquired lipodystrophy are similar to those of congenital lipodystrophy (e.g. insulin resistance diabetes mellitus, hyperinsulinemia, hypertriglyceridemia, and low high-density lipoprotein [HDL] levels). Hepatic involvement is typically more severe in the congenital cases than in the acquired cases, with progression to cirrhosis in $20 \%$ of patients in the former. Hypoglycemic medications, insulin, or metformin may be useful agents to treat lipodystrophy and lower the risk of other metabolic aberrations if diet restriction fails to control serum lipid levels [Herranz et al. 2008].

\section{Nutritional causes}

\section{Total parenteral nutrition}

TPN refers to the intravenous administration of calories and/or glucose. Long-term TPN leads to the depletion of carnitine, a compound necessary for the transfer of free fatty acids from the hepatic cytoplasm into the mitochondria for beta-oxidation. The cytosolic concentration of choline, a critical nutrient for lipoprotein secretion, also decreases, promoting lipid storage in hepatocytes [Allard, 2002]. The duration of TPN administration correlates with the severity of liver injury and in rare cases can result in end-stage liver disease [Cai et al. 2006]. The predominant histologic findings are steatosis, intrahepatic cholestasis, and ductular reduplication (Figure 1). Discontinuation of TPN should be considered in patients who develop abnormal LFTs. 


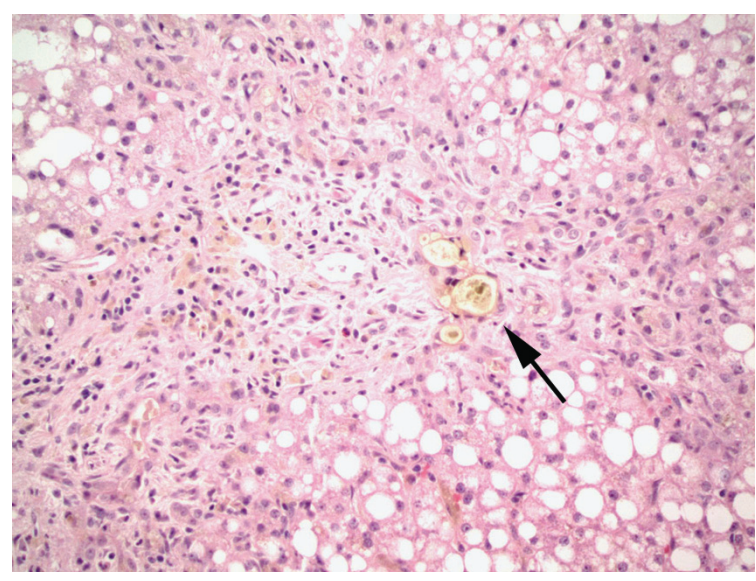

Figure 1. Total parenteral nutrition induced liver injury. The liver shows marked steatosis, cholestasis, and duct proliferation with inspissated bile (arrow).

\section{Severe surgical weight loss}

The now abandoned jejunoileal (JI) bypass procedure carried a $40 \%$ risk of developing abnormal LFTs and $6 \%$ incidence of steatohepatitis. JI bypass leads to the destruction of adipocytes and a tremendous increase in the levels of free fatty acids, which are taken up by the liver [Allard, 2002]. Rarely, Roux-en-Y gastric bypass surgery also results in worsening of steatosis, steatohepatitis, or even cirrhosis [Pillai and Rinella, 2009]. $\mathrm{NASH}$ has also developed after biliopancreatic diversion, extensive small bowel resection, and gastroplasty for morbidly obese patients [Allard, 2002]. These complications run counter to the intuitive assumption that rapid weight loss should normalize metabolic parameters and LFTs. Fortunately, the majority of patients who undergo bariatric surgical procedures show improvement in their steatosis and markers of liver fibrosis. Behavioral modifications such as diet and exercise are appropriate and effective ways to prevent the onset of steatosis after operation.

\section{Starvation}

Severe starvation may lead to steatosis through various mechanisms. Protein deficiency can result in decreased apolipoprotein synthesis, leading to decreased VLDL synthesis and inhibited VLDL transport. VLDL-apolipoprotein B-100 synthesis in protein-energy malnourished children strongly correlates with hepatic fat content [Badaloo et al. 2005]. Although oxidative stress can lead to diminished beta-oxidation of fatty acids, prolonged fasting has actually been associated with
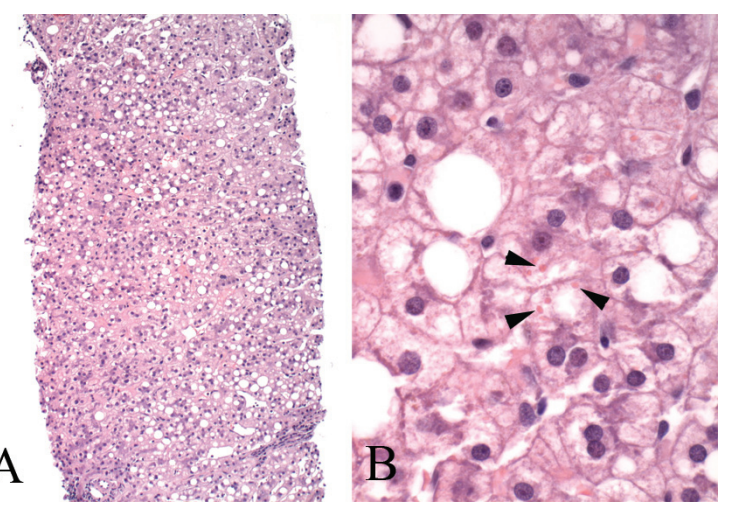

Figure 2. Liver biopsy in a patient on nucleoside analogs. (A) The low-power view shows moderate steatosis in a nonzonal pattern, hepatocyte pallor, and mild hepatic plate thickening suggestive of reactive changes. (B) The high-power view shows the hepatocytes containing numerous swollen 'megamitochondria' (arrowheads), a feature of mitochondrial injury.

higher levels of beta-oxidation. This paradox indicates that reduced VLDL transport plays a more significant role in lipid accumulation during starvation than oxidative stress [Gan and Watts, 2008]. TPN is generally required to ensure sufficient caloric and nutrient intake.

\section{Medications}

\section{HAART}

After the development of antiretroviral therapies to treat HIV disease, the mortality rate due to AIDS decreased while the rate due to liver disease increased. Ultrasonographic evidence of steatosis is found in $31 \%$ of patients with HIV infection. Treatment of patients with HIV using nucleoside reverse transcriptase inhibitors such as didanosine, fialuridine, and zidovudine may induce microvesicular steatosis. Nucleoside analogs lead to hepatotoxicity and steatosis by inhibiting the mitochondrial polymerase-gamma gene and preventing mitochondrial replication, decreasing the net rate of beta-oxidation of fatty acids and facilitating the accumulation of fat [Allard, 2002; CrumCianflone et al. 2009; Price and Thio, 2010]. Protease inhibitors contribute to the development of steatosis by promoting the expression of sterol regulatory element-binding protein 1 (SREBP1), upregulating sterol biosynthesis. Liver histology shows variable steatosis, patchy or extensive microvesicular steatosis, ballooned or clarified hepatocytes, Mallory's hyaline, and occasionally 


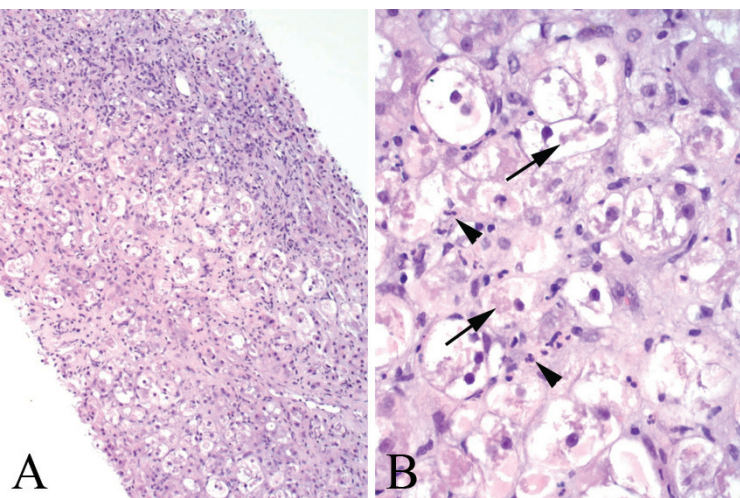

Figure 3. Amiodarone toxicity. (A) Medium-power view shows diffuse ballooning degeneration of hepatocytes and sinusoidal fibrosis. (B) Highpower view shows features reminiscent of alcoholic steatohepatitis, with marked ballooning degeneration of hepatocytes, Mallory-Denk bodies (arrows), and lobular neutrophil infiltrates (arrowheads).

megamitochondria (Figure 2). Management of this condition requires consideration of the need for the antiretroviral therapy, the severity of liver disease, and available alternative therapies that reduce the risk of progressive liver damage while maintaining viral suppression.

\section{Amiodarone}

Nearly a quarter of patients on long-term amiodarone develop mild LFT abnormalities but only $1-3 \%$ have significant inflammation on liver biopsy [Lewis and Zimmerman, 1989]. Amiodarone is associated with a pattern of NASH morphologically similar to alcohol hepatitis. Liver biopsies are characterized by macrovesicular and microvesicular steatosis, ballooning degeneration, neutrophilic infiltration, Mallory-Denk bodies, and sinusoidal fibrosis (Figure 3). A characteristic ultrastructural lesion of amiodarone use is phospholipidosis, in which lysosomes are densely packed with concentric membranous arrays with a fingerprint pattern [Guigui et al. 1988]. Alternative medications should be considered to treat cardiac arrhythmias in patients who suffer from amiodarone-related liver injury.

\section{Tamoxifen}

Tamoxifen (TMX) is associated with the development of steatosis and lobular inflammation and TMX cessation is generally associated with NASH regression [Saphner et al. 2009]. NASH

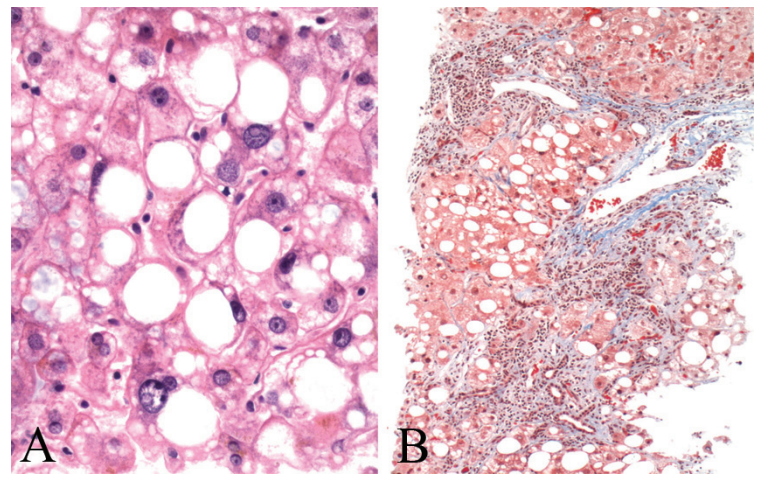

Figure 4. Liver biopsy in the setting of methotrexate. (A) The hepatocytes show macrovesicular steatosis, nuclear hyperchromasia, and anisonucleosis. (B) A trichrome stain shows bridging fibrosis and sinusoidal fibrosis extending from portal tracts.

prevalence in patients taking TMX is highest in those with traditional risk factors for NASH including the metabolic syndrome and obesity. Patients who develop steatosis and abnormal LFTs while on TMX should be assessed for their risk of having NASH (i.e. concurrent metabolic syndrome) and those at high risk for having fibrosis should be referred for liver biopsy, although progression to cirrhosis is rare [Bruno et al. 2005]. In patients found to have advanced fibrosis, the risk of continued TMX use has to be weighed against its benefits.

\section{Methotrexate}

The literature contains many reports of psoriatic patients treated with methotrexate (MTX) who developed cirrhosis. The histologic pattern is that of steatohepatitis, and in this regard, it is interesting that obesity, diabetes, and alcoholism are particular risk factors for methotrexate toxicity. In fact, significant liver injury is uncommon in patients who lack other risk factors of steatohepatitis. Histologic changes include steatosis, ballooning degeneration and necrosis of hepatocytes, nuclear hyperchromasia and pleomorphism, fibrosis with septae extending out from portal tracts, and eventually cirrhosis (Figure 4). Even in patients without evidence of pre-existing NASH, the administration of MTX results in the development of steatosis, inflammation, hepatocyte ballooning degeneration, and fibrosis in a proportion of patients [Langman et al. 2001]. The benefit of MTX treatment should be weighed against the potential for worsening liver disease in patients who develop elevated transaminases while on treatment. 
Table 2. Genetic causes of NAFLD

\begin{tabular}{|c|c|c|c|c|}
\hline Disease & $\begin{array}{l}\text { Genetic } \\
\text { mutation }\end{array}$ & $\begin{array}{l}\text { Age of } \\
\text { presentation }\end{array}$ & Other clinical symptoms & Management \\
\hline Abetalipoproteinemia & $\begin{array}{l}\text { Microsomal } \\
\text { triglyceride } \\
\text { transfer protein }\end{array}$ & Infancy & $\begin{array}{l}\text { Growth problems, mental } \\
\text { retardation }\end{array}$ & $\begin{array}{l}\text { Low-fat diet; fat soluble vitamin } \\
\text { supplementation }\end{array}$ \\
\hline $\begin{array}{l}\text { Familial } \\
\text { hypobetalipoproteinemia }\end{array}$ & ароB100 & Infancy & $\begin{array}{l}\text { Failure to thrive steatorrhea, } \\
\text { spinocerebellar degenerative } \\
\text { ataxia }\end{array}$ & $\begin{array}{l}\text { Low-fat diet; fat soluble vitamin } \\
\text { supplementation }\end{array}$ \\
\hline $\begin{array}{l}\text { Familial combined } \\
\text { hyperlipidemia }\end{array}$ & USF1 & Infancy & $\begin{array}{l}\text { Hypertriglyceridemia, } \\
\text { hypercholesterolemia, }\end{array}$ & $\begin{array}{l}\text { Low-fat diets, exercise, } \\
\text { smoking cessation, weight loss }\end{array}$ \\
\hline $\begin{array}{l}\text { Glycogen storage } \\
\text { disease }\end{array}$ & PHKA2, PHKB & Infancy & $\begin{array}{l}\text { Growth retardation, lactic } \\
\text { acidosis, and development } \\
\text { delay }\end{array}$ & $\begin{array}{l}\text { Avoidance of fasting, } \\
\text { ingestion of corn starch, liver } \\
\text { transplantation }\end{array}$ \\
\hline Weber-Christian disease & unknown & Childhood & $\begin{array}{l}\text { Fever, arthralgias, myalgias, } \\
\text { skin lesions, and painful } \\
\text { subcutaneous nodules }\end{array}$ & $\begin{array}{l}\text { Immunosuppressive reagents, } \\
\text { NSAIDs, glucocorticoids }\end{array}$ \\
\hline $\begin{array}{l}\text { Lipodystrophy } \\
\text { (congenital) }\end{array}$ & AGPAT2, BSCL2 & Infancy & $\begin{array}{l}\text { Severe fat loss, voracious } \\
\text { appetite, accelerated linear } \\
\text { growth, and advanced bone age }\end{array}$ & Low-fat diet \\
\hline
\end{tabular}

\section{Corticosteroids}

Corticosteroids are steroid hormones that are produced by the adrenal cortex and affect cellular change by binding to glucocorticoid receptors within cells. Corticosteroids cause steatosis by inhibiting mitochondrial beta-oxidation and lipid beta-peroxidation enzymes, leading to the accumulation of lipids within hepatocytes. Corticosteroids also induce de novo fatty acid synthesis by activating lipogenic enzymes such as fatty acid synthase, acetyl-CoA carboxylase, and 11 beta-HSD1 in the liver [Jia et al. 2009]. Corticosteroids also contribute to the development of insulin resistance and hyperinsulinemia leading to lipogenesis in the liver. Corticosteroid use in patients with risk factors for NAFLD should be used with caution.

\section{Other causes}

\section{Celiac Disease}

Celiac disease is a systemic immune-related disorder related to gluten sensitivity and accompanied by anti-tissue transglutaminase and anti-endomysial antibodies. Patients with celiac disease often have elevated transaminase levels, and they carry an increased risk of developing fatty liver, hepatitis, fibrosis, and cirrhosis [Ludvigsson et al. 2007]. However, the pathogenesis of fatty liver in celiac disease is not well understood [Abdo et al. 2004]. Adherence to a gluten-free diet is the standard therapy for celiac disease, although its impact on NAFLD has not been well studied.

\section{Wilson disease}

Wilson disease is an autosomal dominant disorder characterized by excess copper deposition in tissues. Wilson disease usually presents in the teenage years or young adulthood with symptoms ranging from neurological dysfunction, chronic liver disease, fulminant hepatic failure, isolated acute hemolysis, to psychiatric illness [McCullough et al. 1983; Steindl et al. 1997]. Wilson disease is caused by mutations in the gene that encodes the copper-transporting ATPase ATP7B [Burkhead et al. 2010]. This mutation prevents copper transport into the Golgi complex and binding of copper to apoceruloplasmin. Copper cannot be excreted in the bile and accumulates in a variety of tissues including hepatocytes. In the liver, the excess copper impairs mitochondrial function and fatty acid beta-oxidation, leading to steatosis. Liver histology is variable and ranges from histologic features indistinguishable from NASH due to metabolic syndrome (Figure 5) to chronic hepatitis due to submassive or massive hepatic necrosis. A copper stain may be helpful, although a negative stain does not exclude the disease and copper quantification may be required for diagnosis. Chelating regimens such as penicillamine, trientine, and zinc are the standard therapy [Farinati et al. 2003]. Liver transplantation may be necessary for a subset of patients. 


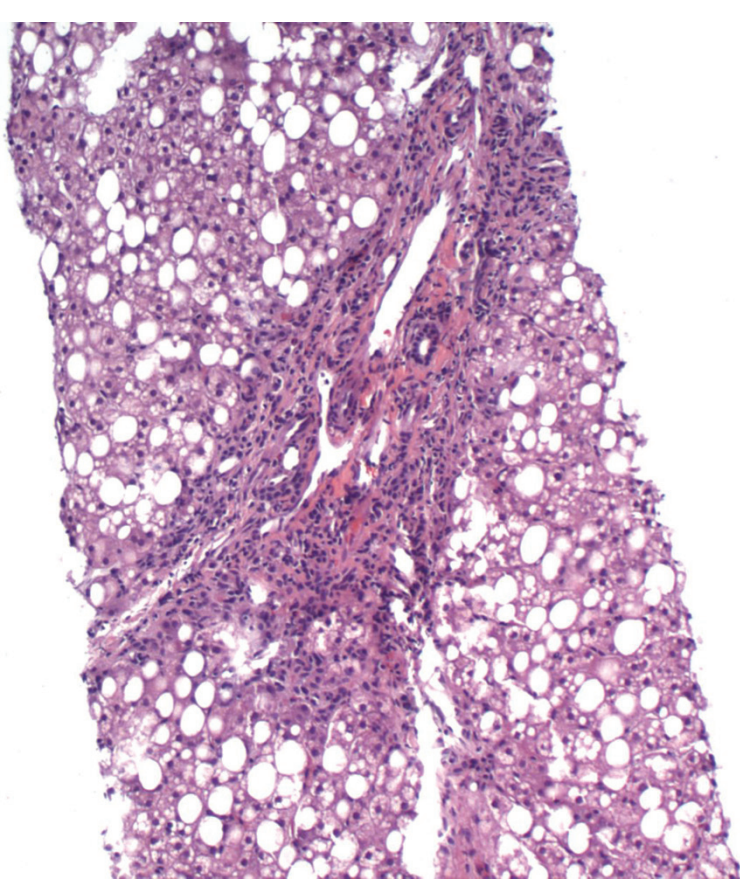

Figure 5. Liver biopsy in a young boy diagnosed with Wilson disease. The biopsy shows features indistinguishable from fatty liver secondary to metabolic syndrome with marked steatosis, irregular portal expansion, and mild portal mononuclear infiltrate. Glycogenated nuclei were also present.

\section{Hepatitis C virus}

Studies have linked hepatitis $\mathrm{C}$ and steatosis, and concurrent hepatitis $\mathrm{C}$ infection and steatosis has been associated with increased risk of disease progression. Genotype 3 is strongly associated with the presence of hepatic steatosis independent of sex, body mass index, and alcohol consumption. Current research suggests that one possible mechanism for hepatic steatosis in HCV is binding of the viral $\mathrm{X}$ gene product to LXR-alpha and upregulation of the transcription factor SREBP1c (sterol regulatory element-binding protein 1c) [Brown, 2008]. Histologically, the steatosis is mild and nonzonal in its distribution unlike the zone 3 accentuation in usual fatty liver disease; however, in cases with concomitant risk factors for NAFLD, the biopsy can have a mixed picture with features of chronic viral hepatitis and steatohepatitis.

\section{Toxicity/environmental}

Rarely, NAFLD has been found in patients exposed to Amanita phalloides mushrooms, phosphorous poisoning, petrochemicals, and Bacillus cereus toxin [Angulo, 2007]. Thus, environmental factors must also be reviewed in the patient history.

\section{Conclusion}

Clinicians are increasingly called upon to diagnose and manage NAFLD. While much of NAFLD can be explained by traditional risk factors of metabolic disease, an important subset are unexplained by these factors and require the clinician to comprehensively evaluate the patient for secondary causes of NAFLD. Identification of these causes is vital for the management of liver disease in these patients and to prevent the accompanying nonhepatic, potentially life-threatening complications of these syndromes. Recent epidemiological studies have found that cholesterol ester storage disease, which is defined by the deficiency in the lysosomal acid lipase (LAL) protein, and Wolman's disease, characterized by the absence of the LAL gene, have lead to steatosis, hepatomegaly, and hypertriglyceridemia and are more common than previously thought [Assman and Seedorf, 2001; Muntoni et al. 2007]. Also less well studied are the neutral lipid storage diseases, defined by the finding of excess triglyceride almost all organs [Igal and Coleman, 1998]. More research is needed to comprehensively identify these causes in order to further strengthen clinicians' toolkit for the diagnosis of NAFLD.

\section{Funding}

This research received no specific grant from any funding agency in the public, commercial, or notfor-profit sectors.

\section{Conflict of interest statement}

The authors declare no conflicts of interest in preparing this article.

\section{References}

Abdo, A., Meddings, J. and Swain, M. (2004) Liver abnormalities in celiac disease. Clin Gastroenterol Hepatol 2: 107-112.

Allard, J.P. (2002) Other disease associations with non-alcoholic fatty liver disease (NAFLD). Best Pract Res Clin Gastroenterol 16: 783-795.

Angulo, P. (2007) GI epidemiology: nonalcoholixc fatty liver disease. Aliment Pharmacol Therapeut 25: 883-889.

Assmann, G., Seedorf, U., Milunsky, A., and Milunsky, K. (2001) Acid lipase deficiency: Wolman disease and cholesterol ester storage disease. In: Sciver, Beuadet, Sly and Valle, The Metabolic and Molecular Bases of Inherited Disease New York: McGraw-Hill, pp. 3551-3572. 
Badaloo, A., Reid, M., Soares, D., Forrester, T. and Jahoor, F. (2005) Relation between liver fat content and the rate of VLDL apolipoprotein B-100 synthesis in children with protein-energy malnutrition. Am f Clin Nutr 81: 1126-1132.

Brown, A.J. (2008) Viral hepatitis and fatty liver disease: how an unwelcome guest makes pate of the host. Biochemical f 416(2): e15-e17.

Bruno, S., Maisonneuve, P., Castellana, P., Rotmensz, N., Rossi, S., Maggioni, M., et al. (2005) Incidence and risk factors for non-alcoholic steatohepatitis: prospective study of 5408 women enrolled in Italian tamoxifen chemoprevention trial. BMF (Clin Res Ed) 330: 932.

Burkhead, J.L., Gray, L.W. and Lutsenko, S. (2010) Systems biology approach to Wilson's disease. Biometals 24(3): 455-456; 575

Burwinkel, B., Rootwelt, T., Kvittingen, E.A., Chakraborty, P.K. and Kilimann, M.W. (2003) Severe phenotype of phosphorylase kinase-deficient liver glycogenosis with mutations in the PHKG2 gene. Pediatric Res 54: 834-839.

Cai, W., Wu, J., Hong, L., Xu, Y., Tang, Q. and Shi, C. (2006) Oxidative injury and hepatocyte apoptosis in total parenteral nutritionassociated liver dysfunction. F Pediatric Surg 41: 1663-1668.

CrumCianflone, N., Dilay, A., Collins, G., Asher, D., Campin, R., Medina, S., et al. (2009) Nonalcoholic fatty liver disease among HIV-infected persons. fAIDS 50: 464-473.

Day, C.P. (2011) Non-alcoholic fatty liver disease: a massive problem. Clin Med 11: 176-178.

de Bruin, T.W.A., Georgieva, A.M., Brouwers, M.C.G.J., Heitink, M.V., van der Kallen, C.J.H. and van Greevenbroek, M.M.J. (2004) Radiological evidence of nonalcoholic fatty liver disease in familial combined hyperlipidemia. Am f Med 116: 847-849.

Farinati, F., Cardin, R., D’Inca, R., Naccarato, R. and Sturniolo, G.C. (2003) Zinc treatment prevents lipid peroxidation and increases glutathione availability in Wilson's disease. $f \mathrm{Lab}$ Clin Med 141: 372-377.

Gan, S.K. and Watts, G.F. (2008) Is adipose tissue lipolysis always an adaptive response to starvation?: implications for non-alcoholic fatty liver disease. Clin Sci 114: 543-545.

Guigui, B., Perrot, S., Berry, J.P., Fleury-Feith, J., Martin, N., Metreau, J.M., et al. (1988) Amiodaroneinduced hepatic phospholipidosis: a morphological alteration independent of pseudoalcoholic liver disease. Hepatology 8: 1063-1068.
Harada, N., Soejima, Y., Taketomi, A., Yoshizumi, T., Uchiyama, H., Ikegami, T., et al. (2009) Recurrent familial hypobetalipoproteinemia-induced nonalcoholic fatty liver disease after living donor liver transplantation. Liver Transplant 15: 806-809.

Herranz, P., de Lucas, R., Pérez-España, L. and Mayor, M. (2008) Lipodystrophy syndromes. Dermatol Clin 26(4).

Igal, R.A. and Coleman, R.A. (1998) Neutral lipid storage disease: a genetic disorder with abnormalities in the regulation of phospholipid metabolism. $\mathcal{F}$ Lipid Res 39: 31-43.

Jia, Y., Viswakarma, N., Fu, T., Yu, S., Rao, M.S., Borensztajn, J., et al. (2009) Conditional ablation of mediator subunit MED1 (MED1/PPARBP) gene in mouse liver attenuates glucocorticoid receptor agonist dexamethasone-induced hepatic steatosis. Gene Expression 14: 291-306.

Kennedy, R.J. and Murphy, L.R. (1949) WeberChristian disease. Am F Med 6: 672-680.

Kishnani, P.S., Austin, S.L., Arn, P., Bali, D.S., Boney, A., Case, L.E., et al. (2010) Glycogen storage disease type III diagnosis and management guidelines. Genetics Med 12: 446-463.

Langman, G., Hall, P.M. and Todd, G. (2001) Role of non-alcoholic steatohepatitis in methotrexateinduced liver injury. $\mathcal{F}$ Gastroenterol Hepatol 16: 1395-1401.

Lewis, J.H. and Zimmerman, H.J. (1989) Druginduced liver disease. Med Clin N Am 73: 775-792.

Linton, M.F., Farese, R.V., Jr and Young, S.G. (1993) Familial hypobetalipoproteinemia. F Lipid Res 34: 521-541.

Ludvigsson, J.F., Elfstrom, P., Broome, U., Ekbom, A. and Montgomery, S.M. (2007) Celiac disease and risk of liver disease: a general population-based study. Clin Gastroenterol Hepatol 5: 63-69.e1.

McCullough, A.J., Fleming, C.R., Thistle, J.L., Baldus, W.P., Ludwig, J., McCall, J.T., et al. (1983) Diagnosis of Wilson's disease presenting as fulminant hepatic failure. Gastroenterology 84: 161-167.

Muntoni, S., Wiebusch, H., Jansen-Rust, M., Rust, S., Seedorf, U., Schulte, H., et al. (2007) Prevalence of cholesteryl ester storage disease. Arterioscler Thromb Vasc Biol 27: 1866-1868.

Muraca, M., Gerunda, G., Neri, D., Vilei, M.T., Granato, A., Feltracco, P., et al. (2002) Hepatocyte transplantation as a treatment for glycogen storage disease type 1a. Lancet 359: 317-318.

Neuschwander-Tetri, B.A. (2005) Nonalcoholic steatohepatitis and the metabolic syndrome. Am F Med Sci 330: 326-335. 
Pillai, A.A. and Rinella, M.E. (2009) Non-alcoholic fatty liver disease: is bariatric surgery the answer? Clin Liver Dis 13: 689-710.

Price, J.C. and Thio, C.L. (2010) Liver disease in the HIV-infected individual. Clin Gastroenterol Hepatol 8: 1002-1012.

Ratziu, V., Bellentani, S., Cortez-Pinto, H., Day, C. and Marchesini, G. (2010) A position statement on NAFLD/NASH based on the EASL 2009 special conference. F Hepatol 53: 372-384.

Reddy, S.K., Kishnani, P.S., Sullivan, J.A., Koeberl, D.D., Desai, D.M., Skinner, M.A., et al. (2007) Resection of hepatocellular adenoma in patients with glycogen storage disease type Ia. f Hepatol 47: 658-663.

Rubenfire, M., Brook, R.D. and Rosenson, R.S. (2010) Treating mixed hyperlipidemia and the atherogenic lipid phenotype for prevention of cardiovascular events. Am F Med 123: 892-898.

Sanyal, A.J., Brunt, E.M., Kleiner, D.E., Kowdley, K.V., Chalasani, N., Lavine, J.E., et al. (2011)
Endpoints and clinical trial design for nonalcoholic steatohepatitis. Hepatology 54: 344-353.

Saphner, T., Triest-Robertson, S., Li, H. and Holzman, P. (2009) The association of nonalcoholic steatohepatitis and tamoxifen in patients with breast cancer. Cancer 115: 3189-3195.

Steindl, P., Ferenci, P., Dienes, H., Grimm, G., Pabinger, I., Madl, C., et al. (1997) Wilson's disease in patients presenting with liver disease: A diagnostic challenge. Gastroenterology 113: $212-218$.

Wasserman, J.M., Thung, S.N., Berman, R., Bodenheimer, H.C., Jr and Sigal, S.H. (2001) Hepatic Weber-Christian disease. Seminars Liver Dis 21: 115-118.

Zeissig, S., Dougan, S.K., Barral, D.C., Junker, Y., Chen, Z., Kaser, A., et al. (2010) Primary deficiency of microsomal triglyceride transfer protein in human abetalipoproteinemia is associated with loss of CD1 function. $\mathcal{F}$ Clin Investigation 120: 2889-2899.
Visit SAGE journals online http://tag.sagepub.com

@SAGE journals 